\title{
The limits of sèlective attention in DAF shadowing
}

\author{
DAVID O. ROBINSON $+\dagger$ \\ University of Oxford, Oxford, England
}

Twelve naive Ss read a passage of English prose at their own speed, and this was tape-recorded and used as the irrelevant message in a dichotic-listening shadowing task. The primary message to which the Ss attended was 1,500 words long, and after the 1,000 th word, the irrelevant message was switched noiselessly to delayed auditory feedback (DAF). Finally, Ss were asked to read a 1,000-word passage while experiencing DAF. The results confirm previous findings that the limits of selective attention are exceeded when DAF is the nonattended message and suggest the importance of immediate auditory feedback in a speech-production mechanism that involves specific delays within the system which are associated with a normal speaking rate for each individual.

In shadowing (Cherry, 1953), the simultaneous repetition of a spoken message, Ss attend to only one message and can ignore one or more irrelevant channels. When Ss are asked to speak or read while receiving DAF of about $200 \mathrm{msec}$, they slow down and make a large number of speech errors (Fairbanks, 1955; Yates, 1963). Two previous experiments (Yates, 1965; Carey, 1969) have attempted to investigate whether the focusing of attention in the dichotic shadowing situation is sufficient to block out the effects of DAF. The results of both studies implied that DAF could not be ignored, but the present experiment was devised to provide a more conclusive test. This study differs considerably in that the Ss had no previous experience of either DAF or shadowing. They had to learn to shadow against an irrelevant message that was recorded in their own voices-this is the only acceptable control condition and was not used in the earlier experiments-and they were not expecting DAF and, hence, were not expecting to be disrupted. The high error rates previously reported for DAF shadowing may have been a result of relatively short passages; the Ss might have had difficulty with a short passage but might perform much better with a longer passage, when the effects of context would

*Preparation of this manuscript was supported by the Department of Defense, Project THEMIS Contract (DAAD05-68-C-0176), to the Institute for the Study of Cognitive Systems through the Texas Christian University Research Foundation.

*The author gratefully acknowledges the advice of Professor Selby H. Evans, Texas Christian University, and Mr. D. Gerver, University of Durham.

$\dagger \dagger$ Requests for reprints should be sent to: ISCS, P.O. Box 30791, TCU, Fort Worth, Texas 76129. be established. In the present experiment, Ss were in the process of shadowing while ignoring their own voices when the irrelevant message became DAF; the study investigated whether Ss could be induced to ignore DAF as well as they could ignore other irrelevant messages in the dichotic-listening shadowing task.

\section{SUBJECTS}

The Ss were 12 male undergraduates with normal hearing; all Ss were naive to the purpose of the experiment, had not had any experience of shadowing or DAF, and had not taken part in any dichotic-listening experiment.

\section{EQUIPMENT}

The equipment consisted of two stereo

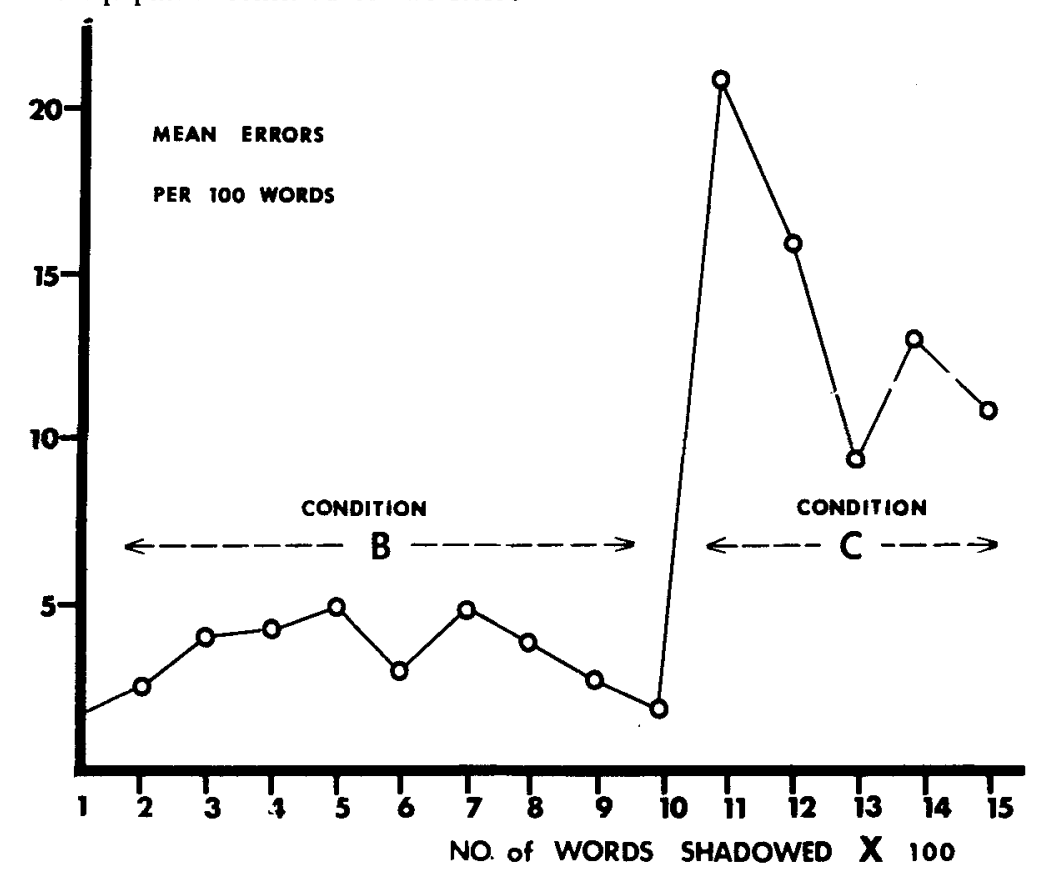

Fig. 1. Mean percentage of errors per 100 words of primary message over all Ss for

Condition B (simple dichotic shadowing) and Condition C (DAF shadowing).

tape recorders (TR1, TR2), two headphone sets, two microphones, and a stereo amplifier, arranged so that two messages could be presented dichoticallv to the $\mathrm{S}$ from TR1. These were monitored by the E; one of the two channels could be either a prerecorded message or DAF. TR2 recorded simultaneously the S's shadowing response on one channel and the primary message on the other.

\section{MATERIAL}

Three passages of narrative English prose without proper nouns were chosen from different sources of approximately the same level of complexity. The primary message was 1,500 words long and was recorded by a male speaker, without errors. Two other passages, one about 1,400 words long and the other about 1,000 words, were to be read aloud by the $S$ under different conditions ( $A$ and $D$, below), and these were typed clearly and without errors on stiff paper.

\section{PROCEDURE}

Each of the Ss underwent the same experimental conditions, and each was tested individually.

$$
\text { Condition A }
$$

The $S$ was seated in a soundproof booth and was handed the typed sheets of the first experimental passage. $S$ was told that he was going to be asked to read the passage aloud in a normal speaking voice but that to begin with he was to look through the piece, paying particular attention to the transitions between pages, so that the passage could be read without a break. When the $S$ said that he had had 


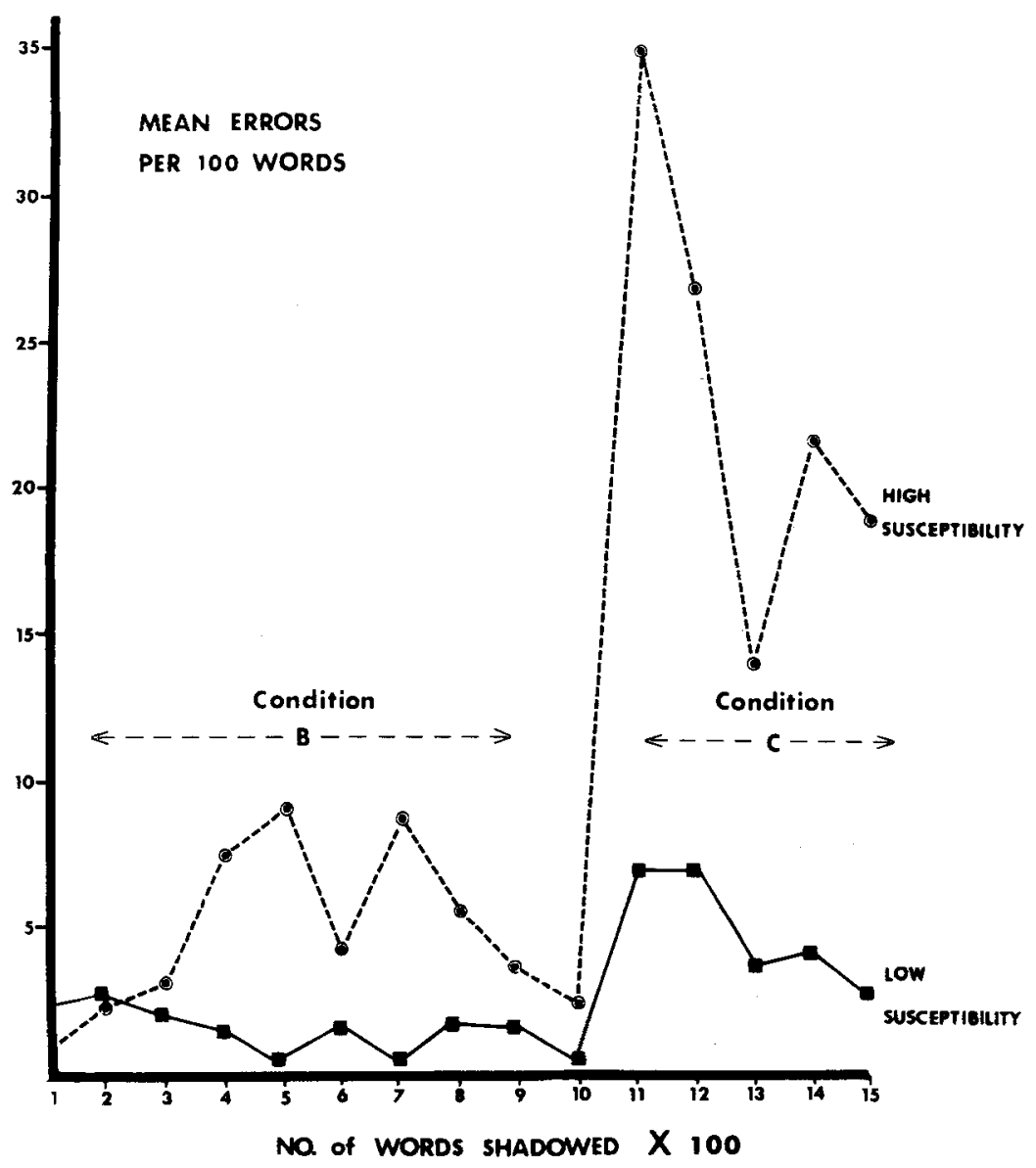

Fig. 2. Mean percentage of shadowing errors per 100 words of primary message for all Ss assigned to two groups on the basis of susceptibility to DAF measured under Condition D (DAF reading).

what he considered to be sufficient time, he read the passage as instructed, and this reading was recorded on the second channel of the master tape (i.e., the tape that had the primary message already recorded on Channel 1). The secondary channel was arranged to start a few seconds after the primary and was faded in over the first few words. By watching the VU meter, the $\mathrm{E}$ attempted to achieve a constant recording level and a similar intensity for all Ss.

\section{Condition B}

The script of the first passage was removed from the booth and the master tape was rewound. The instructions continued, "This is the main part of the experiment. It involves listening to a message over headphones and repeating it back straight away, and is known as shadowing." The E then explained conversationally in detail what was required, that shadowing was a continuous process, and a demonstration was given. When the $\mathrm{S}$ said that he understood the task, it was impressed upon him that it was important to keep going and that if he made a mistake, he should not attempt to go back, but just carry on where the primary message was at that moment. S was told that there would be two messages, one to each ear, but that he was to attend only to the right ear. The $S$ was told that the other channel would be another message, in his own voice, but that he was to ignore this. "Don't be concerned about anything which you may hear in your left ear; don't be distracted from your task, which is to attend to the right ear and shadow that message."

The primary and secondary volumes were adjusted at the S's headphones so that a Dawe sound-level meter showed them to be equal to within $5 \mathrm{~dB}$ of each other (Moray, 1958), with a mean level on the order of $65 \mathrm{~dB}$ (decibel levels relative to 0.002 dynes $/ \mathrm{cm}^{2}$ ). With both tape recorders operating (TR1 "feeding" the S and TR2 recording), the E monitored the messages presented to $S$.

\section{Condition $\mathrm{C}$}

When Word 1,000 of the primary message had been played, TR1 was switched without stopping to record on
Channel 2, in addition to replaying both channels. This had the etfect of instantaneously and noiselessiy switching the left (nonattended) ear input from an irrelevant message to DAF. As the $S$ spoke louder. in an attempt to mask the delayed feedback, the $\mathrm{E}$ attenuated the microphone input to Channel 2 of TR1, maintaining a more or less constant VU reading. When the $S$ had completed the shadowing part of the experiment, a brief rest was permitted. Condition D

In exactly the same way as in Condition $\mathrm{A}$. the $\mathrm{S}$ was asked to look through and then read aloud another passage. the only difference being that in this case the $S$ wore headphones and, as for Condition $C$, the left channel was DAF, with the right headphone being in place but effectively disconnected. SCORING

The following measures were taken. (1) Speed of normal reading: the extent to which each $S$ is disrupted by DAF may be a function of the $S$ s normal speaking rate, and this was estimated by noting the time $S$ took to read passages of known length under Condition A, giving a mean speed of reading in words per minute (wpm). This was compared with a similar measure: (2) speed of reading under DAF. (3) Misarticulations: The number of errors per 100 words of primary message was measured under three conditions--shadowing against own-voice irrelevant message, shadowing against DAF, and reading against DAF. Three types of errors were distinguished: (a) omissions, (b) errors within words, (c) errors within words which were then self-corrected. (4) Ear-voice span: When Ss are accurately and confidently shadowing, they follow the primary message very closely, sometimes as little as one syllable behind. By means of the two-track recording on TR2, the ear-voice span under the two conditions was measured by counting the number of words spoken by the $\mathrm{S}$ between the end of hearing a particular syllable and the start of the S's response to that syllable. This measure was made for 10 words chosen at random in the primary message, for each of the two shadowing conditions for each $S$.

\section{RESULTS}

In all the measures taken, there was a wide range of individual differences (which was expected, since the most disruptive delay for each $S$ is critical and the delay chosen, about $200 \mathrm{msec}$, is a mean critical value).

None of the Ss broke down completely when DAF was introduced or had been conscious of any "click" on the nonattended channel. When shadowing against DAF, the Ss' strategies included 
slowing down and raising the level of the voice.

\section{Articulation}

Figure 1 shows the mean number of errors by all Ss per 100 words of the 1,500-word primary message and clearly shows the markedly larger number of errors made under Condition $\mathrm{C}$ as compared to Condition B. A one-tail $t$ test shows the poorer articulation after Word 1,000 to be significant, $p<.05$ $(t=1.914, \mathrm{df}=11)$. The rise in errors was mainly an increase in the number of omissions rather than within-word errors.

Figure 2 shows that the rise in the mean number of errors was not due simply to a few highly susceptible Ss. The data from all Ss have been split up into two equal groups on the basis of the number of errors made under Condition D (DAF reading). Those who made the most errors constituted the high-susceptibility group, and, conversely, those who were less affected by DAF made up the low-susceptibility group. Although the differences in error scores for the two groups is considerable, the inability to completely ignore DAF-even for Ss of low-susceptibility-is shown.

\section{Latency}

The ear-voice lag did not increase significantly when the irrelevant message was changed to DAF. The mean latency over 1,000 words of simple dichotic shadowing was about 1.5 words, compared with Treisman \& Geffen's (1967) figure of 3.1 words. This difference might have occurred because the present experiment involved intrinsic practice by virtue of the long passages or because Treisman's Ss had additional attentional loads.

\section{Correlation of Variables}

There were strong correlations between the three error rates for each $S$ under Condition $B$ (simple dichotic shadowing), Condition C (DAF shadowing), and Condition D (DAF reading). Ear-voice latency during DAF shadowing was correlated with the error rate (rho $=0.69$, $\mathrm{p}<.02$ ).

\section{CONCLUSIONS}

It appears impossible for Ss to erect a "Broadbent filter" which would enable them to ignore the speech-disruptive effects of DAF. Carey (1969) has suggested that filtering theories and, in particular, filter attenuation are untenable in the light of such data, but Treisman's model would allow for this result. Treisman (1961) proposed that nonattended channels are "attenuated" ("that is, made less easily discriminable") but are nonetheless facilitated at the dictionary stage of the model if they are "probable, "important," or recently received." DAF would be covered by this last category because the $\mathrm{S}$ monitors the direct auditory feedback, and it is this processing that the DAF confuses, causing the speech disruption. It has yet to be shown that direct auditory feedback is essential for speaking; these results are consistent with the view that all speech production tasks require some attention to the immediate feedback and with the view that each individual has a speech production-perception system of which the feedback system is relevant to a normal speaking rate.

\section{REFERENCES}

CAREY, P. W. Delayed auditory feedback and the shadowing response. Unpublished $\mathrm{PhD}$ dissertation. Harvard University, 1969.

CHERRY, E. C. Some experiments on the recognition of speech with one and with two ears. Journal of the Acoustical Society of America, 1953, 25, 975-979.

FAIRBANKS, G. Selective vocal effects of delayed auditory feedback. Journal of Speech \& Hearing Disorders, 1955, 20, 333-346.

MORAY, N. Effect of relative intensities of messages in dichotic shadowing. Language \& Speech, 1958, 1, 110-113.

TREISMAN, A. M. Attention and speech. Unpublished PhD dissertation. Oxford University, 1961.

TREISMAN, A. M., \& GEFFEN, G. Selective attention: Perception or response? Quarterly Joumal of Experimental Psychology, 1967, 19, 1-16.

YATES, A. J. Delayed auditory feedback. Psychological Bulletin, 1963, 60, 213-251.

YATES, A. J. Delayed auditory feedback and shadowing. Quarterly Journal of Experimental Psychology, 1965, 17, 125-131.

\title{
Reinforcement of verbal behavior by evaluative meaning words
}

\author{
CLAIRE F. ETAUGH, RICHARD J. KELLIHER, and RICHARD B. STALLING \\ Bradley University, Peoria, Ill. 61606
}

This study tested the generality of an earlier finding that evaluative meaning words function as reinforcers for children's instrumental motor learning. College students served as $\mathrm{Ss}$ in a verbal conditioning task. Words rated as having positive, negative, or neutral evaluative meaning (EM) were presented contingent upon the use of self-referent pronouns and were later removed during extinction. Frequency of emission of self-referent pronouns: (1) increased, following presentation of positive EM words and removal of negative EM words; (2) decreased, following removal of positive, and presentation of negative, EM words; (3) was unaffected by presentation or removal of neutral EM words. The results strengthen Staats's (1968) conclusion that EM words can function as reinforcers and punishers in instrumental tasks.

Staats (1968) has suggested that the evaluative portion of a word's meaning (Osgood, Suci, \& Tannenbaum, 1957) is established through classical conditioning, and that words which have acquired evaluative meaning will function in instrumental conditioning as reinforcers and punishers. Using sixth-grade children as Ss, Finley \& Staats (1967) demonstrated the reinforcing function of evaluative meaning (EM) words in a button-pushing task; response-contingent positive EM words strengthened the motor response, negative EM words decreased it, and neutral words had no effect on response frequency.

It follows from Staats's position that evaluative meaning words should serve as reinforcers for a wide variety of instrumental behaviors and $S$ populations. The generality of this hypothesis was examined in the present study in a verbal conditioning task with adult Ss.

In addition, while Finley \& Staats (1967) demonstrated that presentation of EM words affects acquisition of an instrumental behavior, the effect of terminating such presentations has not been explored. The present study sought to determine if termination of EM word presentations would produce response extinction, as does the removal of more conventional reinforcers.

\section{SUBJECTS}

Forty-five male undergraduates at Bradley University were assigned randomly 